\title{
129 Middle third facial fractures
}

A Le Fort I (Guerin) involves the orbit.

$B$ Malocclusion is common.

C Trismus is due to associated temporomandibular joint dislocation.

D Epiphora indicates involvement of the nasolacrimal duct.

E An opaque antrum on X-ray is an indication for antral puncture and lavage.

\section{Blowout fracture of the orbit}

A Is caused by excessive force blowing the nose.

B The eyeball herniates into the antrum.

C The patient is unable to look down.

D Treatment should be delayed until oedema has settled if the forced duction test shows limitation of movement.

E Silastic sheeting is a suitable material for repair of the orbital floor.

\section{Fractures involving the frontal or ethmoidal sinuses}

A CSF rhinorrhoea implies a dural tear.

B Nose blowing may cause an intracranial aerocoele.

C Systemic antibiotics are routinely given to prevent meningitis.

D If the posterior wall of the frontal sinus alone is involved, and there is no aerocoele, early repair is unnecessary.

E A fascial graft may be used to repair a dural tear.

\section{Cerebrospinal fluid rhinorrhoea}

A The usual symptom is clear watery fluid dripping from the nose.

$B$ The fluid contains glucose.

C The site of the leak is determined by clinical examination.

D Initial treatment is to pack the nose with BIPP.

E A lumboperitoneal shunt should be considered before resorting to craniotomy. 